\title{
ANÁLISE DE FERRAMENTAS COMPUTACIONAIS UTILIZADAS EM MODELOS NUMÉRICOS PARA REPRESENTAÇÃO DE PROCESSOS ATMOSFÉRICOS, OCEÂNICOS E DE SUPERFÍCIE
}

\author{
Eliseo Breda Silva ${ }^{1}$, Dirceu Herdies ${ }^{2}$ and Mario Francisco Leal De Quadro ${ }^{3}$
}

\begin{abstract}
Resumo
A transformação digital é uma realidade em todas as áreas da atividade humana e vem sendo acelerada pela recente pandemia da Covid-19. O uso de Inteligência Artificial, Data Analytics, Realidade Aumentada, Robots, dentre outras tecnologias viabiliza a automação de processos praticamente em todos os segmentos da economia: agricultura, manufatura e serviços. Entender os fenômenos climáticos e seus impactos no ambiente sempre foi um desafio para o homem que, desde a antiguidade e até os dias atuais, se depara com situações não previstas, que trazem impacto em seu dia a dia, colocando, muitas vezes, a própria vida em risco. Na atualidade, além de buscar entender, o que se busca é prever o clima e seus impactos ambientais, de modo a mitigar os riscos, viabilizar a continuidade do desenvolvimento, preservando a natureza (fauna, flora, recursos naturais etc). O uso de ferramentas computacionais para modelagem numérica que representem este fenômenos é de suma importância, endereçando a necessidade de previsões cada vez mais rápidas e confiáveis. Este projeto analisa ferramentas disponíveis no mercado, que utilizam três modelos numéricos distintos, descrevendo as funcionalidades existentes e a assertividade na previsão de fenômenos climáticos em Santa Catarina.
\end{abstract}

\section{Palavras-chave}

NWP, acuracidade, modelos

\section{INTRODUÇÃO}

O uso de ferramentas computacionais para modelagem numérica que representem os processos atmosféricos, oceânicos e de superfície é de suma importância, permitindo a simulação de cenários ambientais e identificação de tendências, com rapidez e segurança. $O$ aumento da capacidade computacional tornou mais fácil o uso destas ferramentas, com redução significativa dos tempos de processamentos, contribuindo, dessa maneira, para a popularização no uso de modelos numéricos de previsão do tempo, que inicialmente era muito concentrado em centros operacionais como, por exemplo, o National Center for Environmental Prediction (NCEP). Com isso, começaram a ser adotados também por universidades, agências governamentais e a iniciativa privada (MASS e KUO, 1998) durante os últimos 10 anos.

Nesse sentido, os modelos meteorológicos de mesoescala têm sido utilizados como ferramentas de pesquisa para melhorar o entendimento dos processos de meso escala e para o desenvolvimento e aprimoramento das parametrizações destes processos para uso em modelos de previsão climática de larga escala. Três modelos meteorológicos de meso escala são muito conhecidos entre os especialistas: MM5 (Mesoscale Model - fifth Generation), COAMPS (Coupled
Ocean / Atmospheric Mesoscale Prediction System) e WRF (Weather Research and Forecasting).

A avaliação da performance e acuracidadedestes modelos é fator crítico para a melhoria e entendimento dos mesmos, tendo como base seus pontos fortes e fracos. Isto permite obter importantes informações para o aprimoramento da acuracidade dos modelos e traz significado para as previsões. Assim, este trabalho tem o objetivo de avaliar os resultados de diferentes modelos numéricos utilizados na previsão de volumes de precipitação, temperatura do ar, umidade do ar, velocidade e direção dos ventos, radiação e ondas oceânicas, no estado de Santa Catarina, comparando-os com os dados observados e calculando os desvios entre

${ }^{1}$ Mestrando em Clima e Ambiente, Instituto Federal de Santa Catarina, Florianópolis, Santa Catarina

${ }^{2}$ Mestrado em Clima e Meio Ambiente - IFSC, Instituto Nacional de Pesquisas Espaciais

${ }^{3}$ Instituto Federal de Santa Catarina, Florianópolis, Santa Catarina Email: dherdies@gmail.com. (Dirceu Herdies)

Corresponding author:

Eliseo Breda Silva, Mestrando em Clima e Ambiente, Instituto Federal de Santa Catarina, Florianópolis, Santa Catarina

Email: eliseo.breda.silva@gmail.com. 
eles. Busca-se com isto, gerar indicadores objetivos que auxiliem os profissionais da área de meteorologia na escolha de modelos mais assertivos, a depender dos processos que estiverem estudando. Em particular, será avaliada a acuracidade dos modelos nas previsões dos eventos extremos de precipitação, ondas de calor / frio, vendavais e ressacas oceânicas ocorridos na região nos últimos cinco anos.

\section{REVISÃO BIBLIOGRÁFICA}

Muitos estudos avaliativos de verificação destes modelos tem sido realizados, a maioria dos quais, focados no modelos de mesoescala de quinta geração do National Center for Atmospheric Reserach (NCAR) da Universidade Estadual da Pensilvânia (MM5; ) (Grell, Dudhia, \& Stauffer, 1994). Entre eles incluem-se as comparações com outros modelos de várias regiões do mundo, tais como Colle, Westrick, and Mass (1999), Nutter e Manobianco (1999), White et al. (1999), Mass et al. (2002), Hong (2003), e Colle et al. (2003a, b).

O trabalho de White et al. (1999) avaliou seis modelos no oeste dos Estados Unidos durante a estação fria (Janeiro, Fevereiro e Março) e demonstrou que ocorreu variação considerável no viés de cada modelo. O estudo mostrou que os maiores erros ocorreram nos modelos de previsão que apresentavam maior resolução horizontal. Os resultados também mostraram a necessidade de comparações multi modelos, de modo a permitir identificar os erros e vieses relacionados à física dos modelos e os benefícios e pontos fracos em função do aumento da resolução.

Usando o MM5 com resolução horizontal de $4 \mathrm{Km}$, Hanna e Yang (2001) avaliaram a performance do modelo num período de 4 dias, comparando a velocidade e direção do vento observadas a $10 \mathrm{~m}$,acima da superfície do solo comos resultados do modelo. Os resultados mostraram que o erro médio (EM) e a raiz do erro quadrático médio (REQM) do erro da velocidade média horária do vento foram de 1.5 e 2.5 $\mathrm{m} / \mathrm{s}$, respectivamente, para um intervalo de velocidades do vento entre $2.8 \mathrm{~m} / \mathrm{s}$ e $3.4 \mathrm{~m} / \mathrm{s}$. O EM e REQM foram de -2 graus e 66 graus, respectivamente. Eles sugeriram que estas incertezas na velocidade e direção do vento foram causadas principalmente por processos de turbulência randômicos, que não puderam ser simulados pelos modelos, mesmo o com variações nos terrenos e uso da terra.

Muitos estudos também têm focado em comparações entre os modelos de previsão MM5 e NCEP Eta (ONCLEY E DUDHIA 1995; MANNING E DAVIS 1997; ; COLLE et al. 1999, 2000, 2003a; CHENG E STEENBURGH 2005) (White et al., 1999). Oncley e Dudhia (1995) constataram que o aumento excessivo da umidade do solo nas simulações levou a um viés de resfriamento durante o dia,

gerando aumento no fluxo de calor latente (resfriamento evaporativo) e diminuição do calor sensível. Usando o MM5,

Manning e Davis (1997) demonstraram que o acréscimo da porção de gelo nas montanhas, como condição inicial, aprimoraram o viés de frio nas baixas altitudes durante o dia.

No que diz respeito a acuracidade da previsão de precipitação, não se observa uma rápida evolução nos

últimos anos se comparada com a acuracidade de outras variáveis meteorológicas (Olson, Junker, \& Korty, 1995). De acordo com os autores, os erros nas previsões quantitativas da precipitação são resultados principalmente das incertezas relativas as condições iniciais, resolução horizontal insuficiente em regiões de topografia acidentada, além de deficiências nas parametrizações dos modelos no que diz respeito à microfísica e convecção.

Após estudar as previsões de precipitação no oeste dos Estados Unidos utilizando MM5, Colle et al. (1999; 2000) Colle et al. (1999) mostraram que, durante a estação fria, a acuracidade do modelo aumentou com a redução espacial do grid de 36 para $12 \mathrm{~km}$. Entretanto, a redução adicional do grid espacial para $4 \mathrm{Km}$ gerou baixo incremento na acuracidade, exceto para chuvas pesadas, devido a superpredição da precipitação nas partes altas dos terrenos.

Wang e Seaman (1997) chegaram a conclusão semelhante em seus estudos de modelagem para ambos os grids de 12 e $36 \mathrm{~km}$, utilizando o mesmo critério de parametrização convectiva. Eles concluíram que usando a parametrização convectiva com a grade espacial de $12 \mathrm{~km}$ houve melhora na previsão da quantidade de precipitação, o que não ocorreu com a grade espacial de $36 \mathrm{~km}$.

Por outro lado, Gallus (1999) constatou que houve um pequeno benefício na previsão da quantidade de precipitação usando a parametrização convectiva, quando da redução da grid espacial de 78 para $12 \mathrm{~km}$.

\section{METODOLOGIA}

O projeto analisará os três modelos disponíveis no mercado (WRF, Eta e BRAMS), do ponto de vista de funcionalidades existentes e assertividade na previsão de fenômenos climáticos em Santa Catarina. A escolha destes três modelos foi baseada na disponibilidade de dados observados para a área de estudo selecionada.

\section{Metodologia utilizada será a seguinte:}

(i) descrição da documentação técnica disponível e versões para instalação de ferramentas que utilizam os três modelos citados para uso em ambiente de laboratório;

(ii) instalação dos modelos na base computacional do Laboratório de Meteorologia do IFSC;

(iii) realização de testes estatísticos com os dados gerados pelos modelos e, se aplicável, de parametrizações e demais configurações dos modelos para a área selecionada;

(iv) busca na base histórica de informações de clima e fenômenos naturais em nosso estado, que possam ser utilizadas para os testes de uso prático das ferramentas escolhidas.

(v) Avaliação de casos específicos.

\section{CONCLUSÕES}

Conclui-se que existem grandes variabilidades e incertezas no uso dos modelos numéricos para a representação e previsão dos processos atmosféricos, oceânicos e de superfícies, sendo necessária análise detalhada e cuidadosa para escolha do que utilizar, quando utilizar, com que bases de dados, de modo que os resultados obtidos sejam representativos e tenham acuracidade.

O produto final que será um relatório técnico, contendo as funcionalidades existentes em cada ferramenta, procurando 
destacar tanto as disponíveis em todas como as específicas de cada uma, possibilitando uma leitura de que diferenciais existem entre elas.

Também serão apresentados os resultados dos testes práticos realizados, a fim de que seja possível identificar a assertividade de cada ferramenta no tratamento das informações e fenômenos climáticos analisados

\section{REFERÊNCIAS}

\section{References}

(2002).

Cheng, W., \& Steenburgh, W. J. (2005). Evaluation of surface sensible weather forecasts by the wrf and eta models over the western united states (Vol. 20). USA, v: Weather and Forecasting.

Colle, B. A., Mass, C. F., \& Westrick, K. J. (2000).

Colle, B. A., Olson, J. B., \& Tongue, J. S. (2003a). Multiseason verification of the mm5. part $i$ : Comparison with eta model over the central and eastern united states and impact of mm5 resolution (Vol. 18). USA, v: Weather and Forecasting.

Colle, B. A., Olson, J. B., \& Tongue, J. S. (2003b). Multiseason verification of the mm5. part ii: Evaluation of high-resolution precipitation forecasts over the northeastern united states (Vol. 18). USA, v: Weather and Forecasting.

Colle, B. A., Westrick, K. J., \& Mass, C. F. (1999). Evaluation of the $\mathrm{mm} 5$ and eta-10 precipitation forecasts over the pacific northwest during the cool season (Vol. 14). USA, v: Weather and Forecasting.

Gallus, W. A. (1999).

Grell, G. A., Dudhia, J., \& Stauffer, D. R. (1994). A description of the fifth generation Penn State/NCAR Mesoscale Model (MM5). NCAR Tech. Note NCAR/TN-398 1A. National Center for Atmospheric Research, 107-107.

Hanna, S. R., \& Yang, R. (2001). Evaluation of mesoscale models' simulations of near-surface winds, temperature gradients, and mixing depths. Journal of Applied Meteorology and Climatology, 40, 10951104.

Hong, J. S. (2003). (Vol. 18).

Manning, K. W., \& Davis, C. A. (1997). (Vol. 12).

Mass, C. F., Ovens, D., Westrick, K. J., \& Colle, B. A. (n.d.). Does increasing horizontal resolution produce better forecast? The results of two years of real-time numerical weather prediction in Pacific Northwest. Bulletin of the American Meteorological Society.

Nutter, P., \& Manobianco, J. (1999).

Olson, D. A., Junker, N. W., \& Korty, B. (1995). Evaluation of 33 years of quantitative precipitation forecasts at nmc. weather and forecasting. USA, v.10.

Oncley, S. P., \& Dudhia. (1995). Evaluation of surface fluxes from MM5 using observations. Monthly Weather Review, 125, 3344-3357.

Wang, W., \& Seaman, N. L. (1997). A comparison study pf convective parameterization schemes in a mesoscale model. Monthly Weather Review, 125, 252-278.
White, G. B., Paegle, J., Steenburgh, W. J., Horel, J. D., Swanson, R. T., Cook, L. K., . . Miles, J. G. (1999). Short-term forecast validation of six models (Vol. 14). USA, v: Weather and Forecasting. 